\title{
Differential circulating concentrations of adipokines, glucagon and adropin in a clinical population of lean, overweight and diabetic cats
}

Rizaldy C. Zapata ${ }^{1 *+}$, Melissa D. Meachem²†, Natalia Cavalca Cardoso ${ }^{3}$, Susan O. Mehain ${ }^{4}$, Chantal J. McMillan ${ }^{5}$, Elisabeth R. Snead ${ }^{3}$ and Prasanth K. Chelikani ${ }^{1}$

\begin{abstract}
Background: Dyslipidemia, dysregulated adipokine secretion and alteration in glucagon and adropin concentrations are important obesity-related factors in the pathophysiology of human Type 2 diabetes; however, their roles in the pathophysiology of feline diabetes mellitus are relatively unknown. Here, we determined the concentrations of circulating leptin, adiponectin, pro-inflammatory cytokines, glucagon, adropin, triglycerides, and cholesterol, in non-diabetic lean and overweight cats and newly diagnosed diabetic cats. Client-owned cats were recruited and assigned into 3 study groups: lean, overweight and diabetic. Fasting blood samples were analyzed in lean, overweight and diabetic cats at baseline and 4 weeks after consumption of high protein/low carbohydrate standardized diet.

Results: Serum concentrations of triglycerides were greater in diabetics at baseline and were increased in both diabetic and overweight cats at 4 weeks. Plasma leptin concentrations were greater in diabetic and overweight at baseline and 4 weeks, whereas adiponectin was lower in diabetics compared to lean and overweight cats at baseline and 4 weeks. Diabetics had greater baseline plasma glucagon concentrations compared to lean, lower adropin than overweight at 4 weeks, and lower IL-12 concentrations at 4 weeks than baseline.

Conclusions: Our results suggest that feline obesity and diabetes mellitus are characterized by hypertriglyceridemia and hyperleptinemia; however, diabetic cats have significantly lower adiponectin and adropin compared to overweight cats. Thus, despite having similar body condition, overweight and diabetic cats have differential circulating concentrations of adiponectin and adropin.
\end{abstract}

Keywords: Feline diabetes, Obesity, Adipokines, Glucagon, Adropin

\section{Background}

Diabetes mellitus is a common endocrinopathy in cats [1-3]; however, its pathophysiology is not completely understood. Similar to humans with Type 2 diabetes mellitus (T2DM), obesity is a common clinical feature of feline diabetes mellitus (FDM) [4-6]. Increasing body weight has clearly been shown to decrease insulin sensitivity in cats supporting the important link between

\footnotetext{
*Correspondence: rczapata@ucalgary.ca

${ }^{\dagger}$ Equal contributors

'Department of Production Animal Health, University of Calgary, 3280

Hospital Drive NW, Calgary, AB T2N4N1, Canada

Full list of author information is available at the end of the article
}

obesity and glucose homeostasis [7, 8]. Dyslipidemia, dysregulated adipokine secretions and alteration in glucagon and adropin concentrations are important obesity-related factors in the pathophysiology of human T2DM [9, 10]; however, their roles in the pathophysiology of FDM are relatively unknown [11].

Adipose tissue is a source of hormones and cytokines that modulate energy balance and glucose metabolism. There is substantial evidence that obese and insulinresistant cats have increased circulating concentrations of leptin when compared to healthy lean cats $[12,13]$. However, the association of adiponectin with obesity is 
inconsistent in felines with some studies indicating a negative correlation [14-18] and others failing to detect a relationship between total circulating adiponectin and obesity $[19,20]$. In addition, the adipose transcript abundance of pro-inflammatory cytokines (eg., tumor necrosis factor- $\alpha$, interferon- $\Upsilon$, monocyte chemoattractant protein-1) has been reported to be greater in obese compared to lean cats [21-23]. However, less is known of whether circulating concentrations of these inflammatory mediators are altered with adiposity and diabetes in cats. Moreover, obese cats have increased plasma triglyceride concentrations [24] and higher cholesterol concentrations have been associated with a decreased likelihood of diabetic remission in FDM [25].

The role of gut hormones in the pathophysiology of feline obesity and diabetes is poorly understood. We recently reported that plasma concentrations of the gut hormone glucagon-like peptide-1 (GLP-1) are increased after a meal in cats, with diabetic cats having greater circulating concentrations of GLP-1 than lean or overweight cats [26]. Although the preproglucagon gene encodes for GLP-1 in the gut and glucagon in the pancreas [27], the mechanisms of glucagon secretion and action in FDM are poorly understood. There is some evidence that diabetic cats that underwent remission had a higher glucagon to insulin ratio [28] and that humans with T2DM have hyperglucagonemia [10]. However, whether this dysregulation in glucagon secretion is because of obesity and/or diabetic state is relatively unknown in felines. In addition to the gut, the liver is also being increasingly recognized as an important source of hormones that regulate energy and glucose homeostasis. For example, adropin, a relatively newly discovered peptide hormone, is synthesized and secreted primarily by the liver but is also found in the heart and brain [9]. Dietary macronutrients stimulate adropin secretion and systemic injections of adropin to obese mice have been shown to improve glucose tolerance, skeletal muscle insulin sensitivity and promote weight loss [9, 29]. The role of adropin in the pathophysiology of feline obesity and diabetes is unknown. Therefore, the objective of the present study was to determine whether there were differences in the concentrations of circulating triglycerides, cholesterol, leptin, adiponectin, cytokines, glucagon and adropin in lean, overweight and diabetic cats.

\section{Methods}

Experimental protocols, subject descriptions, feeding protocols, and health assessments have been previously reported for this cohort of cats as part of a study to measure plasma concentrations of GLP-1, glucosedependent insulinotropic peptide (GIP), insulin and peptide YY (PYY) in client-owned newly diagnosed diabetic cats, and non-diabetic lean or overweight cats [26]. The data on circulating metabolites and hormones that we now provide in the current study have not been previously reported elsewhere.

Briefly, the experimental protocols (AC13-0197) were approved by the University of Calgary Animal Care and Use Committee and the Western College of Veterinary Medicine Animal Research Ethics Board and were conducted in compliance with each university's ethical guidelines for animal research. Thirty-one cats were recruited from the University of Calgary Veterinary Medicine/Western Veterinary Specialists and Emergency Centre and the Veterinary Medical Center at Western College of Veterinary Medicine through a request for participation. Inclusion criteria were based on body condition score (BCS), the absence of concurrent disease or lack of any medication and acceptance of restraint, venipuncture and standardized diets while exclusion criteria included those that were not amenable to restraint and venipuncture, poor appetites, current history of chronic vomiting or diarrhea, presence of concurrent disease including renal disease or hyperthyroidism, and any current medication administration for any endocrine disorders including, but not limited to, treatment for hyperthyroidism or any medications known to influence glycemia (i.e. glucocorticoids).

\section{Demographics}

The demographics of the study population have been previously reported [26]. Briefly, healthy cats with a BCS of $\leq 5$ were categorized as lean (LC) and those with $\mathrm{BCS} \geq 6$ as overweight (OC) based on the 9-point BCS [30]. The BCS was assessed independently by experienced investigators (authors CJM, ERS). A total of 10 healthy lean and 11 overweight domestic shorthairs were enrolled in the study. LC included 5 neutered males and 5 spayed females while OC included 6 neutered males and 5 neutered females. Mean age was $8.7 \pm 1.1$ years in LC and $6.63 \pm 0.8$ years in OC. Mean body weight was $4.4 \pm 0.2 \mathrm{~kg}$ in $\mathrm{LC}$ and $6.3 \pm 0.3 \mathrm{~kg}$ in $\mathrm{OC}$ while mean BCS was 5 in LC and 7 in OC. Ten diabetic (DC) cats were enrolled in this study wherein 8 were neutered males while 2 were spayed females. Diabetic cats were diagnosed based on client history and clinical signs, hyperglycemia with concurrent glucosuria and elevated fructosamine. DC had a mean age of 10.6 (range 5-15) years, body weight of $5.7 \pm 0.5 \mathrm{~kg}$ and BCS of 7 (3 lean, 7 overweight).

\section{Feeding protocol}

The feeding protocol was described in full detail previously [26]. Briefly, to eliminate the effects of varying nutrition contents of different feline diets, LC and OC were fed a standardized diet composed of a combination of dry (Adult Optimal Care ${ }^{\circledast}$ Hill's Science Diet ${ }^{\oplus}$, 
Topeka, KS) and canned (Adult Gourmet Turkey Entrée, Hill's Science Diet $\left.{ }^{\circ}\right) 2$ weeks prior to the baseline hormone assessments. Following the baseline assessment, LC and OC were exclusively fed a combination of dry and canned high protein diet commonly prescribed to diabetic cats (Prescription Diet ${ }^{\circ} \mathrm{m} / \mathrm{d}^{\circ}$, Hill's Science Diet $^{\circ}$ ) until the 4-week assessment. To avoid multiple dietary changes in a feline patient with a newly diagnosed chronic illness, DC were not transitioned to the standardized diet and had baseline assessments performed while on the diets being fed at the time of diagnosis and were then immediately transitioned to the same diabetic diet fed to $\mathrm{LC}$ and $\mathrm{OC}$ and started on exogenous insulin therapy.

\section{Blood sampling and health assessment}

Blood collection was performed as described previously [26]. Briefly, sampling was performed after a 10-h fast in LC and OC after 2 weeks on the standardized diet (baseline) and then at 4 weeks on the diabetic diet. In DC, blood was also collected after a 10-h fast at baseline and then at 4 weeks on the diabetic diet. For all animals, a complete history was taken at each assessment point including owners' compliance with diet and fasting recommendations. At the time of each evaluation, animals had a complete physical examination which included body weight and BCS assessments.

\section{Diabetic management and insulin therapy}

Insulin therapy was carried out as previously reported [26]. Briefly, DC were treated with glargine (Lantus ${ }^{\circ}$, Sanofi-Aventis, Laval, QC) twice a day at starting dose of $0.25 \pm 0.02 \mathrm{U} / \mathrm{kg}$ during the morning and $0.23 \pm 0.02 \mathrm{U} / \mathrm{kg}$ in the evening - with dosages adjusted upon the recommendation of the attending clinician. DC were evaluated routinely for ongoing management of their FDM, however, only preselected time points were used to collect study data. At each recheck, a thorough history was taken. Owners were specifically questioned about clinical signs that could be used as subjective measures of improvement in hyperglycemia, including decreases in polyuria, polydipsia, and polyphagia. DC also had blood glucose curve performed and serum fructosamine concentrations measured at each sampling point. Clinical signs, physical examination parameters, and blood glucose assessments were used to make therapeutic decisions regarding insulin dosage adjustments.

\section{Measurement of plasma hormone and metabolite concentrations}

Fasting plasma concentrations of leptin, adiponectin, glucagon and adropin were measured at baseline and at 4 weeks using commercially available ELISA kits. Only fasting samples were analyzed because it has previously been shown that postprandial concentrations of leptin and adiponectin in cats are not statistically different from fasting samples [31]. Similarly, since glucagon concentrations are highly influenced by circulating insulin concentrations [10], with special consideration to our insulin-treated DC, only fasting blood samples are analyzed. In this case, exogenous insulin would not have been administered to DC for $12 \mathrm{~h}$ prior to sampling. In addition, only fasting adropin concentrations were measured as it has been reported in humans that adropin concentrations do not dramatically change after consumption of high sugar or fat meals [32]. Plasma concentrations IL-1b, IL-6, IL-12, MCP-1, and TNF $\alpha$ were determined using Milliplex ${ }^{\circ}$ ELISA by an independent laboratory (Eve Technologies, Calgary, AB). Each sample was assayed in duplicate following the manufacturer's recommended protocols. All assays underwent validation procedures using pooled cat plasma. Fasting serum triglyceride, cholesterol, liver enzymes and other analytes (Alkaline phosphatase (ALP), Alanine aminotransferase (ALT), Gamma-glutamyl transferase (GGT), Glutamate dehydrogenase (GLDH) and other analytes (Blood urea nitrogen (BUN), albumin, bilirubin) and fructosamine concentrations were measured using standard laboratory protocols at Prairie Diagnostic Services (Saskatoon, SK).

To minimize the effect of inter-assay variability, the samples were distributed so that each plate received an approximately equal number of samples from each treatment group. Inter-assay $\mathrm{CV}$ was assessed by running pooled cat plasma in duplicate on each plate.

Leptin was measured at baseline and at the 4-week assessment point using a feline leptin-specific assay with a range of $0-125 \mathrm{nmol} / \mathrm{L}$ (FEE0445, Biotang, Lexington, MA). The intra-assay and interassay CV's were 12 and $24 \%$, respectively and the assay sensitivity was $30 \mathrm{pmol} /$ L. Spikes of 15.6 and $31.3 \mathrm{nmol} / \mathrm{L}$ of leptin in pooled cat plasma yielded recoveries of 101 and $122 \%$, respectively.

Adiponectin was measured at baseline and at the 4 week assessment point with an assay that cross-reacts with human, canine, hamster, monkey, feline and rat adiponectin; with a range of 3-694 nmol/L (RD191023100, BioVendor, Brno, Czech Republic), and was previously validated for feline samples [19]. The intra-assay CV for was $5 \%$, interassay $\mathrm{CV}$ was $24 \%$, and assay sensitivity was $3 \mathrm{nmol} / \mathrm{L}$. Spikes of $30 \mathrm{nmol} / \mathrm{L}$ to pooled cat plasma yielded a recovery of $100 \%$.

Glucagon concentration was measured using an assay that cross-reacts with human, rat and mouse glucagon with a range of 1.5-120 pmol/L (10-1271-01, Mercodia, Uppsala, Sweden). The intra-assay CV was 5.6\%; interassay CV was $3.2 \%$, and assay sensitivity was $1.7 \mathrm{pmol} / \mathrm{L}$. Spikes of 9.06 and $30.5 \mathrm{pmol} / \mathrm{L}$ of glucagon to pooled 
cat plasma resulted in 101 and 105\% recoveries, respectively. Linear regression of expected versus measured concentrations for glucagon from serially diluted plasma (1:2 to $1: 8)$ in independent assays yielded average slope of 1.07, $R^{2}$ values $(P<0.001)$ of 0.99 , and Y-intercept of -3.36 .

Adropin concentration was measured using an assay that cross-reacts with human, mouse and rat adropin, and has a range of 2-20,000 pmol/L (EK-032-35, Phoenix Pharmaceuticals, Burlingame, CA). The intra-assay CV was $14.0 \%$, interassay CV was $0.9 \%$ and assay sensitivity was $652 \mathrm{pmol} / \mathrm{L}$. Spikes of 200 and 2000 of adropin to pooled cat plasma yielded recoveries of 86 and $82 \%$, respectively. Linear regression of expected versus measured concentrations for adropin in serially diluted (1:2 to $1: 8)$ pooled cat plasma yielded average slope of $0.66, R^{2}$ values $(P<0.001)$ of 0.92 and $Y$-intercept of 23.10 .

Plasma concentrations of IL-1b, IL-6, IL-12, MCP-1 and TNF $\alpha$ were measured using a customized feline cytokine assay that utilizes Multiplexing LASER Bead Technology. The intra-assay CV's, inter-assay CV's and recoveries for IL-1b were 10,35 and $32 \%$, respectively, IL-6 were 28, 28 and 34\%, respectively, IL-12 were 5, 9 and $112 \%$, respectively, MCP-1 were 31,35 and $42 \%$, respectively and TNF $\alpha$ were 20,52 and $32 \%$, respectively. Data for IL-1b, IL-6, MCP-1 and TNF $\alpha$ were not considered for further analyses due to poor assay performance. Only data for IL-12 were analyzed and reported.

\section{Statistics}

Data on LC, OC and DC are reported as mean \pm SE and were analyzed using IBM SPSS ${ }^{\oplus}$ v20 (New York, USA). The data for all hormones were log-transformed prior to analyses to improve normality and then analyzed by repeated measures linear mixed models using age and gender as covariates, group (LC, OC, and DC) as between subject factor, week (baseline and 4 week) as within group factor, and group $\times$ week interaction. Age and gender were subsequently removed from the overall model as they were not significant. Cats within the group were the random variable on which repeated measures were taken and covariance structures modeled. The covariance structure of the repeated measurements for each variable was modeled either as compound symmetry, heterogeneous compound symmetry, autoregressive, heterogeneous autoregressive, first order antedependence or Toeplitz based on the smallest values of fit statistics for Akaike's and Bayesian information criteria. The between-group differences for each week were analyzed using ANCOVA with age and gender as covariates followed by Bonferroni test to separate means. The within-group differences between baseline and 4-week hormone concentrations were analyzed with paired $t$-test. Pearson correlation analyses were done to assess the relationship among variables. Significance was set at $P \leq 0.05$ and trends at $P<0.10$.

\section{Results}

\section{Body weight and BCS}

As we previously reported [26], at 4 weeks, the OC $(6.4 \pm 0.3 \mathrm{~kg})$ and $\mathrm{DC}(5.8 \pm 0.5 \mathrm{~kg})$ were significantly heavier than LC $(4.4 \pm 0.2 \mathrm{~kg})$. Further, the $\mathrm{OC}$ $(7.0 \pm 0.2)$ and DC $(7.0 \pm 0.8)$ had greater BCS than LC $(5 \pm 0.1)$ at 4 weeks.

\section{Glucose, insulin, triglycerides, cholesterol and liver enzymes}

Fasting blood glucose and plasma insulin were previously reported [26] and are provided here together with serum liver enzymes, BUN, albumin and bilirubin in Table 1. For serum triglyceride concentrations (Fig. 1a), the main effect of group was significant $(P<0.05)$, group $\mathrm{x}$ week interactions $(P=0.09)$ tended to be significant, whereas week $(P=0.21)$ was not significant. At baseline, DC had greater triglyceride concentrations by 117 and 96\% compared to LC and OC, respectively. At 4 weeks, triglycerides were significantly increased in $\mathrm{OC}$ and DC by 63 and 73\% compared to LC, respectively.

For cholesterol concentrations (Fig. 1b), the main effect of group $(P=0.17)$ and group $\mathrm{x}$ week interactions $(P=0.22)$ were not significant, whereas week $(P<0.01)$ was significant. From baseline to 4 weeks within groups, the transition to the standardized diet commonly prescribed to diabetic patients resulted in significantly increased circulating cholesterol concentrations in LC by $11 \%$, OC by $27 \%$ and in DC by $13 \%$.

\section{Leptin, adiponectin, and IL-12}

For plasma leptin concentrations (Fig. 1c), the main effect of group was significant $(P<0.01)$, whereas week $(P=0.70)$ and group $\mathrm{x}$ week interactions $(P=0.98)$ were not. At baseline, leptin concentrations in $\mathrm{OC}$ and $\mathrm{DC}$ were increased by 172 and $221 \%$ compared to LC, respectively. At 4 weeks, plasma leptin concentrations in OC and DC were increased by 188 and $214 \%$ compared to LC, respectively. Overall, leptin concentrations were positively correlated with BCS $(r=0.43, P<0.01)$ and triglycerides $(r=0.29, P=0.02)$.

The main effect of group was significant $(P=0.01)$ for plasma adiponectin (Fig. 1d), with a trend for week $(P=0.07)$ but no significant group $\mathrm{x}$ week interaction $(P=0.81)$. At baseline, DC had 61 and $45 \%$ lower adiponectin than LC and OC, respectively. At 4 weeks, circulating concentrations of adiponectin were significantly lower in DC by 81 and 69\% compared to LC and OC, respectively. Overall, adiponectin concentrations were negatively correlated with BCS $(r=-0.29, P=0.03)$ and triglycerides $(r=-0.32, P=0.02)$.

No significant difference was observed in the plasma concentrations of IL-12 (Fig. 1e) across groups $(P=0.87)$. There was a trend for group $\mathrm{x}$ week 
Table 1 Fasting blood glucose, plasma insulin and serum liver enzymes concentrations in lean, overweight and newly-diagnosed diabetic cats at baseline

\begin{tabular}{lllll}
\hline & Reference & Lean & Overweight & Diabetic \\
\hline Glucose $(\mathrm{mmol} / \mathrm{L})$ & $3.5-8.1$ & $3.99 \pm 0.16$ & $4.45 \pm 0.46$ & $21.07 \pm 1.25$ \\
Insulin (pmol/L) & $\mathrm{N} . \mathrm{A}$. & $65.20 \pm 16.43$ & $80.92 \pm 23.58$ & $47.38 \pm 15.20$ \\
${ }^{\mathrm{a}} \mathrm{ALP}(\mathrm{U} / \mathrm{L})$ & $11-56$ & $27.12 \pm 2.15$ & $24.82 \pm 2.20$ & $55.70 \pm 5.56$ \\
${ }^{\mathrm{b}} \mathrm{ALT}(\mathrm{U} / \mathrm{L})$ & $30-120$ & $66 \pm 6.82$ & $63.09 \pm 7.96$ & $90.60 \pm 25.43$ \\
${ }^{\mathrm{c}} \mathrm{GGT}(\mathrm{U} / \mathrm{L})$ & $0-6$ & $0.25 \pm 0.16$ & $0 \pm 0$ & $0.20 \pm 0.13$ \\
${ }^{\mathrm{d}} \mathrm{GLDH}(\mathrm{U} / \mathrm{L})$ & $1-5$ & $2 \pm 0.37$ & $2.64 \pm 0.43$ & $23.56 \pm 14.65$ \\
${ }^{\mathrm{e}} \mathrm{BUN}(\mathrm{mmol} / \mathrm{L})$ & $6-11.4$ & $8.77 \pm 0.89$ & $9.74 \pm 0.53$ & $8.30 \pm 0.56$ \\
Albumin $(\mathrm{g} / \mathrm{L})$ & $27-39$ & $37.88 \pm 1.57$ & $38.09 \pm 0.80$ & $37.90 \pm 1.15$ \\
Bilirubin $(\mu \mathrm{mol} / \mathrm{L})$ & $0-2$ & $1.35 \pm 0.35$ & $1.49 \pm 0.22$ & $1.53 \pm 0.15$ \\
\hline
\end{tabular}

Values are expressed as mean \pm SEM, $n=10-11 /$ group

${ }^{a}$ Alkaline phosphatase

${ }^{\mathrm{b}}$ Alanine aminotransferase

'Gamma-glutamyl transferase

${ }^{\mathrm{d}}$ Glutamate dehydrogenase

e Blood Urea nitrogen

interaction $(P=0.07)$ with plasma IL-12 concentrations decreasing by $25 \%$ from baseline to 4 weeks in DC.

\section{Glucagon and adropin}

There was a significant group effect for circulating glucagon (Fig. 1f) concentrations $(P=0.01)$ but none for week $(P=0.14)$ and group $\mathrm{x}$ week interactions $(P=0.12)$. At baseline, DC had increased glucagon compared to LC by $236 \%$ but not compared to OC. No differences were observed at 4 weeks. Glucagon concentrations were positively correlated with triglycerides $(r=0.30, P=0.03)$ and cholesterol ( $r=0.26, P=0.05)$.

For plasma adropin concentrations (Fig. 1g), there were significant effects of group $(P=0.03)$ and group $\mathrm{x}$ week interactions $(P<0.01)$. At baseline, no difference was observed among groups. However, at 4 weeks, OC had increased adropin concentrations compared to LC and DC by 112 and 79\%, respectively. From baseline to 4 weeks within each group, adropin was significantly increased by $35 \%$ in OC but tended to be decreased by $34 \%$ in LC. Overall, adropin concentrations were positively correlated with $\mathrm{BCS}(r=0.27 P=0.05)$.

\section{Discussion}

We compared the circulating concentrations of triglycerides, cholesterol, leptin, adiponectin, IL-12, glucagon and adropin in client-owned lean, overweight and diabetic cats. Our results highlight several important findings. First, serum concentrations of triglycerides were greater in diabetic at baseline and were increased in both diabetic and overweight cats at 4 weeks. Second, plasma leptin concentrations were greater in diabetic and overweight cats at baseline and 4 weeks, whereas adiponectin was lower in diabetic compared to lean and overweight cats at baseline and 4 weeks after starting the cats on the high protein/low carbohydrate standardized diet. Third, diabetic cats had greater baseline plasma glucagon concentrations compared to lean cats, lower adropin than overweight cats at 4 weeks, and lower IL-12 concentrations at 4 weeks than baseline.

The hypertriglyceridemia in the $\mathrm{OC}$ and DC is consistent with other reports demonstrating that increased circulating triglycerides are seen in obese [24] but not lean cats [33]. Similar to humans, plasma lipids are also elevated in T2DM [34]. Feeding the commercially available high protein and low carbohydrate diet commonly prescribed to diabetics did not affect triglycerides but increased cholesterol in all groups. To our knowledge, the effect of high protein/low carbohydrate diets on cholesterol in $\mathrm{OC}$ and $\mathrm{DC}$ are not yet reported. In kittens, high protein/low carbohydrate diets did not change cholesterol concentrations [35]. Cholesterol also did not differ between healthy adult cats fed with a moderate protein/high carbohydrate diet and high protein/low carbohydrate diet at comparable fat contents [36]. Because the dietary macronutrient composition of the $\mathrm{m} / \mathrm{d}$ diet in our study is in general comparable to other reports, it is less likely that the increased cholesterol in $\mathrm{OC}$ and DC are primarily of dietary origin. However, whether the dietary factors interact with the host to predispose $\mathrm{OC}$ and DC to enhanced cholesterogenesis remain to be determined.

The circulating leptin concentrations reflected adiposity and were significantly elevated in DC and OC compared with LC at baseline and 4 weeks. Our results are in agreement with other studies supporting the correlation with adiposity with increased leptin concentrations in overweight and obese cats and increases in cats undergoing weight gain [19, 37]. Similar to other studies [31, 38], fasting leptin concentrations were also not affected by feeding 

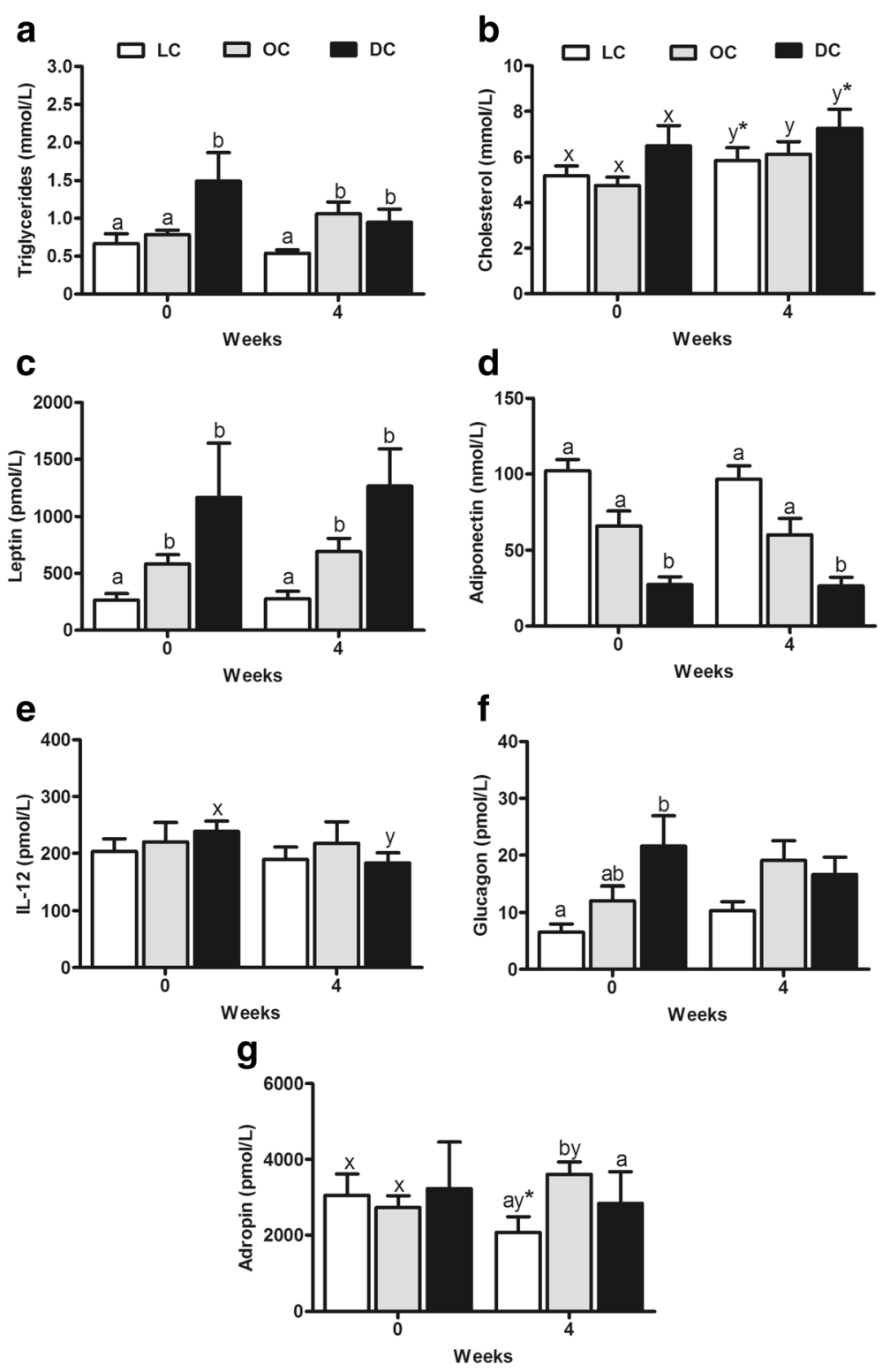

Fig. 1 Concentrations of serum triglycerides (a), cholesterol (b), and plasma leptin (c), adiponectin (d), IL-12 (e), glucagon (f) and adropin (g) in lean $(n=10)$, overweight $(n=11)$ and diabetic $(n=10)$ cats at baseline and after 4 weeks of feeding a commonly prescribed feline diabetic diet. a,b denotes differences between groups at each week; $x, y$ denotes differences between baseline and 4 weeks $(P<0.05)$. ${ }^{*}$ denotes a trend $(P<0.10)$. Values are mean \pm SEM

a high protein diet which is likely due to the maintenance of a stable body weight in our subjects.

We found that DC had significantly decreased adiponectin compared to OC and LC throughout the study. The effects of adiposity on circulating total adiponectin concentrations are controversial with some studies indicating that $\mathrm{OC}$ have decreased adiponectin concentrations [14-18], whereas others failed to detect differences between LC and OC in total adiponectin $[19,20]$. In our study, total adiponectin concentrations across all groups showed weak negative correlation with BCS. Similarly, in humans with T2DM, adiponectin concentrations are greatly reduced compared to obese humans [39]. Importantly, we also noted a reduction of adiponectin concentrations in DC compared to both LC and OC, suggesting that decreased adiponectin is also present in FDM. The lower adiponectin concentrations in DC might have contributed to the lower negative correlation of BCS with adiponectin despite comparable BCS between the $\mathrm{OC}$ and $\mathrm{DC}$.

Previous rodent $[40,41]$ and human $[42,43]$ studies reported that pro-inflammatory cytokine IL-12 is 
increased during obesity and T2DM; however, there is limited data in cats. Hoenig et al. [21, 44] reported an increase in the mRNA abundance of TNF- $\alpha$ in white adipose but without differences in plasma concentrations of TNF- $\alpha$, IL-1, and IL- 6 of OC compared to LC. Van de Velde et al. [22] reported increased mRNA abundance of TNF- $\alpha$, IFN- $\Upsilon$, chemokine ligand 5 (CCL-5) and MCP-1 in adipose tissue of $\mathrm{OC}$ compared to $\mathrm{LC}$ but did not detect differences in IL-6 and IL-10. It is unclear whether the alterations in mRNA abundance of cytokines in adipose tissues are reflective of circulating cytokine concentrations in cats. In our study, despite the feline-specific nature of the multiplex assay for cytokines, the poor assay performance $(<50 \%$ recoveries) of IL-1b, IL-6, MCP-1, and TNF $\alpha$ precluded their assessment in our patient population. However, the recoveries and assay CV's for IL-12 were acceptable. We noted that IL-12 did not differ between OC and LC but decreased in DC. Given that IL-12 secretion is increased in T2DM [40-43], whether the reduction in IL-12 in DC at 4 wks is due to improvements brought about by exogenous insulin administration or dietary change from baseline remains to be addressed.

At baseline, prior to exogenous insulin therapy, DC had greater plasma glucagon concentrations compared to LC. Previous research did not find a statistical difference in the number of immunoreactive $\alpha$-cells between DC and non-DC [23] but Tschour et al. [28] reported a higher ratio of glucagon to insulin in FDM that remitted versus those that did not. In humans, increased circulating concentration of glucagon is an indicator of insulin resistance and diabetic status [45]. Our results also support the hypothesis that glucagon is involved in FMD pathophysiology; whether the lack of differences in plasma glucagon concentrations at 4 weeks is a consequence of an insulin-induced downregulation of glucagon secretion from exogenous insulin therapy in DC remains to be demonstrated.

For the first time, we report on plasma concentrations of adropin in cats. At baseline, adropin concentrations did not differ between our study groups. However, we found that adropin concentrations were increased in OC but not LC or DC following 4 weeks. The association of adropin with diabetes is controversial. In rodents, increased adropin immunoreactivity was noted particularly in the pancreas, liver and kidneys of streptozotocin diabetic rats [46], whereas adropin concentrations were found to be lower in women with gestational diabetes [47-49] and exogenous adropin improves glucose tolerance and insulin action in mice [29]. Interestingly, in our study, despite comparable BCS and diets, adropin concentrations were lower in DC than OC; whether this is due to diabetic status and/or exogenous insulin therapy remains to be determined.

\section{Conclusions}

Our results suggest that overweight and diabetic cats are characterized by hypertriglyceridemia and hyperleptinemia; however, reduced adiponectin and adropin in diabetics are disassociated from overweight cats. Thus, feline obesity and diabetes are associated with differential circulating concentrations of adiponectin and adropin. Whether the differential circulating concentrations of these hormones are due to dysregulations in the tissue expression, secretion, action and/or elimination remain to be determined in cats with varying degrees of body condition and diabetic status.

\section{Abbreviations \\ ALP: Alkaline phosphatase; ALT: Alanine aminotransferase; ANCOVA: Analysis of covariance; BCS: Body condition score; BUN: Blood urea nitrogen; CCL- 5: Chemokine ligand 5; CV: Coefficient of variation; DC: Diabetic cats; \\ ELISA: Enzyme-linked immunosorbent assay; FDM: Feline diabetes mellitus; GGT: Gamma-glutamyl transferase; GIP: Glucose-dependent insulinotropic peptide; GLDH: Glutamate dehydrogenase; GLP-1: Glucagon-like peptide-1; IFNY: Interferon gamma; IL-12: Interleukin-12; IL-1B: Interleukin-1B; IL- 6: Interleukin-6; LC: Lean cats; MCP-1: Monocyte chemoattractant protein-1; OC: Overweight cats; PYY: Peptide YY; T2DM: Type 2 diabetes mellitus; TNFa: Tumor necrosis factor alpha}

\section{Acknowledgement}

The authors thank Justin Haskett, Dr. llona Houston and Dr. Kevin Cosford for their contributions with the patient recruitment and sample collection.

\section{Funding}

This work was supported by the University of Calgary Clinical Research Fund to CJM, NSERC Discovery Grant (355993-2013) to PKC, and the University of Saskatchewan to ERS.

\section{Availability of data and materials}

The datasets used and/or analyzed during the current study is available from the corresponding author on reasonable request.

\section{Authors' contributions}

CJM, ERS and PKC designed the study. MDM, NCC, SOM, CJM and ERS recruited patients and performed animal experiments. RCZ performed all hormone assays and statistical analyses. RCZ and MDM drafted the manuscript. NCC, SOM, CJM, ERS and PKC edited the manuscript. All authors read and approved the final manuscript.

\section{Competing interests}

The authors declare that they have no competing interests.

Consent for publication

Not applicable.

\section{Ethics approval}

The experimental protocols were approved by the University of Calgary Animal Care and Use Committee and the Western College of Veterinary Medicine Animal Research Ethics Board and were conducted in compliance with each university's ethical guidelines for animal research. Prior to recruitment, all cat owners were required to sign a consent form outlining the details, as well as the potential risks and benefits of this study.

\section{Publisher's Note}

Springer Nature remains neutral with regard to jurisdictional claims in published maps and institutional affiliations.

\section{Author details}

${ }^{1}$ Department of Production Animal Health, University of Calgary, 3280 Hospital Drive NW, Calgary, AB T2N4N1, Canada. ${ }^{2}$ Department of Veterinary Pathology, University of Saskatchewan, 52 Campus Drive, Saskatoon, SK, 
Canada. ${ }^{3}$ Department of Small Animal Clinical Sciences, Western College of Veterinary Medicine, University of Saskatchewan, 52 Campus Drive, Saskatoon, SK, Canada. ${ }^{4}$ Veterinary Teaching Hospital, College of Veterinary Medicine, Washington State University, 205 Ott Rd, Pullman, WA 99164-7060, USA. ${ }^{5}$ Department of Veterinary Clinical and Diagnostic Sciences, Faculty of Veterinary Medicine, University of Calgary, 3280 Hospital Dr. NW, Calgary, AB, Canada.

\section{Received: 26 November 2016 Accepted: 29 March 2017} Published online: 04 April 2017

\section{References}

1. McCann TM, Simpson KE, Shaw DJ, Butt JA, Gunn-Moore DA. Feline diabetes mellitus in the UK: the prevalence within an insured cat population and a questionnaire-based putative risk factor analysis. J Feline Med Surg. 2007:9:289-99.

2. Prahl A, Guptill L, Glickman NW, Tetrick M, Glickman LT. Time trends and risk factors for diabetes mellitus in cats presented to veterinary teaching hospitals. J Feline Med Surg. 2007:9:351-8.

3. Lederer R, Rand JS, Jonsson NN, Hughes IP, Morton JM. Frequency of feline diabetes mellitus and breed predisposition in domestic cats in Australia. Vet J. 2009:179:254-8

4. Cefalu WT. Animal models of type 2 diabetes: clinical presentation and pathophysiological relevance to the human condition. ILAR J. 2006:47:186-98.

5. Martin G, Rand J. Current understanding of feline diabetes: part 2, treatment. J Feline Med Surg. 2000;2:3-17.

6. O'Brien TD. Pathogenesis of feline diabetes mellitus. Mol Cell Endocrinol. 2002;197:213-9.

7. Osto M, Lutz TA. Translational value of animal models of obesity-focus on dogs and cats. Eur J Pharmacol. 2015;759:240-52.

8. Clark M, Hoenig M. Metabolic effects of obesity and its interaction with endocrine diseases. Vet Clin North Am Small Anim Pract. 2016;46:797-815.

9. Ganesh Kumar K, Zhang J, Gao S, Rossi J, McGuinness OP, Halem HH, Culler MD, Mynatt RL, Butler AA. Adropin deficiency is associated with increased adiposity and insulin resistance. Obesity (Silver Spring). 2012;20:1394-402.

10. Gromada J, Franklin I, Wollheim CB. Alpha-cells of the endocrine pancreas: 35 years of research but the enigma remains. Endocr Rev. 2007;28:84-116.

11. Osto M, Zini E, Reusch CE, Lutz TA. Diabetes from humans to cats. Gen Comp Endocrinol. 2013;182:48-53.

12. Lusby AL, Kirk CA, Bartges JW. The role of key adipokines in obesity and insulin resistance in cats. J Am Vet Med Assoc. 2009:235:518-22.

13. German AJ, Ryan VH, German AC, Wood IS, Trayhurn P. Obesity, its associated disorders and the role of inflammatory adipokines in companion animals. Vet J. 2010;185:4-9.

14. Muranaka S, Mori N, Hatano Y, Saito TR, Lee P, Kojima M, Kigure M, Yagishita $M$, Arai T. Obesity induced changes to plasma adiponectin concentration and cholesterol lipoprotein composition profile in cats. Res Vet Sci. 2011;91:358-61.

15. Hoenig M, Thomaseth $\mathrm{K}$, Waldron $\mathrm{M}$, Ferguson DC. Insulin sensitivity, fat distribution, and adipocytokine response to different diets in lean and obese cats before and after weight loss. Am J Physiol Regul Integr Comp Physiol. 2007;292:R227-34.

16. Ishioka K, Omachi A, Sasaki N, Kimura K, Saito M. Feline adiponectin: molecular structures and plasma concentrations in obese cats. J Vet Med Sci. 2009;71:189-94.

17. Tvarijonaviciute A, German AJ, Martínez-Subiela S, Tecles F, Ceron JJ. Analytical performance of commercially available assays for feline insulin-like growth factor 1 (IGF-1), adiponectin and ghrelin measurements. J Feline Med Surg. 2012;14:138-46.

18. Tvarijonaviciute A, Ceron JJ, Holden SL, Morris PJ, Biourge V, German AJ. Effects of weight loss in obese cats on biochemical analytes related to inflammation and glucose homeostasis. Domest Anim Endocrinol. 2012;42:129-41.

19. Bjornvad CR, Rand JS, Tan HY, Jensen KS, Rose FJ, Armstrong PJ, Whitehead $J P$. Obesity and sex influence insulin resistance and total and multimer adiponectin levels in adult neutered domestic shorthair client-owned cats. Domest Anim Endocrinol. 2014;47:55-64.

20. Witzel AL, Kirk CA, Kania SA, Bartges JW, Boston RC, Moyers T, Byrd H, Lauten S. Relationship of adiponectin and its multimers to metabolic indices in cats during weight change. Domest Anim Endocrinol. 2015:53:70-7.
21. Hoenig M, Pach N, Thomaseth K, Le A, Schaeffer D, Ferguson DC. Cats differ from other species in their cytokine and antioxidant enzyme response when developing obesity. Obesity (Silver Spring). 2013;21:E407-14.

22. Van de Velde $H$, Janssens GP, de Rooster $H$, Polis I, Peters I, Ducatelle $R$, Nguyen P, Buyse J, Rochus K, Xu J, Verbrugghe A, Hesta M. The cat as a model for human obesity: insights into depot-specific inflammation associated with feline obesity. Br J Nutr. 2013;110:1326-35.

23. Zini E, Lunardi F, Zanetti R, Heller RS, Coppola LM, Ferro S, Guscetti F, Osto M, Lutz TA, Reusch CE, Cavicchioli L. Endocrine pancreas in cats with diabetes mellitus. Vet Pathol. 2016;53:136-44.

24. Jordan E, Kley S, Le NA, Waldron M, Hoenig M. Dyslipidemia in obese cats. Domest Anim Endocrinol. 2008;35:290-9.

25. Zini E, Hafner M, Osto M, Franchini M, Ackermann M, Lutz TA, Reusch CE. Predictors of clinical remission in cats with diabetes mellitus. J Vet Intern Med. 2010:24(6):1314-21.

26. McMillan CJ, Zapata RC, Chelikani PK, Snead EC, Cosford K. Circulating concentrations of glucagon-like peptide 1, glucose-dependent insulinotropic peptide, peptide YY, and insulin in client-owned lean, overweight, and diabetic cats. Domest Anim Endocrinol. 2016;54:85-94.

27. Campbell JE, Drucker DJ. Pharmacology, physiology, and mechanisms of incretin hormone action. Cell Metab. 2013;17:819-37.

28. Tschuor F, Zini E, Schellenberg S, Wenger M, Kaufmann K, Furrer D, Lutz TA, Reusch CE. Remission of diabetes mellitus in cats cannot be predicted by the arginine stimulation test. J Vet Intern Med. 2011;25:83-9.

29. Gao S, McMillan RP, Zhu Q, Lopaschuk GD, Hulver MW, Butler AA. Therapeutic effects of adropin on glucose tolerance and substrate utilization in dietinduced obese mice with insulin resistance. Mol Metab. 2015:4:310-24.

30. Laflamme D. Development and validation of a body condition- score system for cats: a clinical tool. Feline Pract. 1997;25:13-8.

31. Coradini M, Rand JS, Morton JM, Arai T, Ishioka K, Rawlings JM. Fat mass, and not diet, has a large effect on postprandial leptin but not on adiponectin concentrations in cats. Domest Anim Endocrinol. 2013:45:79-88

32. Butler AA, St-Onge MP, Siebert EA, Medici V, Stanhope $K L$, Havel PJ. Differential responses of plasma Adropin concentrations to dietary glucose or fructose consumption in humans. Sci Rep. 2015:5:14691.

33. Zini E, Osto M, Konrad D, Franchini M, Sieber-Ruckstuhl NS, Kaufmann K, Guscetti F, Ackermann M, Lutz TA, Reusch CE. 10-day hyperlipidemic clamp in cats: effects on insulin sensitivity, inflammation, and glucose metabolismrelated genes. Horm Metab Res. 2010:42:340-7.

34. Sacks FM, Hermans MP, Fioretto P, Valensi P, Davis T, Horton E, Wanner C, Al-Rubeaan K, Aronson R, Barzon I, Bishop L, Bonora E, Bunnag P, Chuang LM, Deerochanawong C, Goldenberg R, Harshfield B, Hernández C, Herzlinger-Botein $\mathrm{S}$, Itoh $\mathrm{H}$, Jia W, Jiang YD, Kadowaki T, Laranjo N, Leiter L, Miwa T, Odawara M, Ohashi K, Ohno A, Pan C, Pan J, Pedro-Botet J, Reiner Z, Rotella CM, Simo R, Tanaka M, Tedeschi-Reiner E, Twum-Barima D, Zoppini G, Carey VJ. Association between plasma triglycerides and highdensity lipoprotein cholesterol and microvascular kidney disease and retinopathy in type 2 diabetes mellitus: a global case-control study in 13 countries. Circulation. 2014;129:999-1008.

35. Vester BM, Belsito KR, Swanson KS. Serum metabolites, ghrelin and leptin are modified by age and/or diet in weanling kittens fed either a high- or moderate-protein diet. Anim Sci J. 2012:83:426-33.

36. Dobenecker B, Kienzle E, Sallmann HP, Fuhrmann H. Effect of diet on plasma triglycerides, cholesterol, beta-hydroxybutyrate and free fatty acids in cats. J Nutr. 1998;128:2648S-50S.

37. Appleton DJ, Rand JS, Sunvold GD. Plasma leptin concentrations in cats: reference range, effect of weight gain and relationship with adiposity as measured by dual energy X-ray absorptiometry. J Feline Med Surg. 2000;2 191-9.

38. Wei A, Fascetti AJ, Liu KJ, Villaverde C, Green AS, Manzanilla EG, Havel PJ, Ramsey JJ. Influence of a high-protein diet on energy balance in obese cats allowed ad libitum access to food. J Anim Physiol Anim Nutr 2011:95:359-67.

39. Weyer C, Funahashi T, Tanaka S, Hotta K, Matsuzawa Y, Pratley RE, Tataranni PA. Hypoadiponectinemia in obesity and type 2 diabetes: close association with insulin resistance and hyperinsulinemia. J Clin Endocrinol Metab. 2001; 86:1930-5.

40. Wen Y, Gu J, Li SL, Reddy MA, Natarajan R, Nadler JL. Elevated glucose and diabetes promote interleukin-12 cytokine gene expression in mouse macrophages. Endocrinology. 2006;147:2518-25. 
41. Nam H, Ferguson BS, Stephens JM, Morrison RF. Impact of obesity on IL-12 family gene expression in insulin responsive tissues. Biochim Biophys Acta. 1832;2013:11-9.

42. Wegner M, Winiarska H, Bobkiewicz-Kozłowska T, Dworacka M. IL-12 serum levels in patients with type 2 diabetes treated with sulphonylureas. Cytokine. 2008:42:312-6.

43. Suárez-Álvarez K, Solís-Lozano L, Leon-Cabrera S, González-Chávez A, Gómez-Hernández G, Quiñones-Álvarez MS, Serralde-Zúñiga AE, HernándezRuiz J, Ramírez-Velásquez J, Galindo-González FJ, Zavala-Castillo JC, De León-Nava MA, Robles-Díaz G, Escobedo G. Serum IL-12 is increased in Mexican obese subjects and associated with low-grade inflammation and obesity-related parameters. Mediat Inflamm. 2013;2013:967067.

44. Hoenig M, McGoldrick JB, de Beer M, Demacker PN, Ferguson DC. Activity and tissue-specific expression of lipases and tumor-necrosis factor alpha in lean and obese cats. Domest Anim Endocrinol. 2006;30:333-44.

45 Shah P, Basu A, Basu R, Rizza R. Impact of lack of suppression of glucagon on glucose tolerance in humans. Am J Phys. 1999;277:E283-90.

46 Aydin S, Kuloglu T, Aydin S, Eren MN, Yilmaz M, Kalayci M, Sahin I, Kocaman $\mathrm{N}$, Citil C, Kendir Y. Expression of adropin in rat brain, cerebellum, kidneys, heart, liver, and pancreas in streptozotocin-induced diabetes. Mol Cell Biochem. 2013;380:73-81.

47 Celik E, Yilmaz E, Celik O, Ulas M, Turkcuoglu I, Karaer A, Simsek Y, Minarec $Y$, Aydin S. Maternal and fetal adropin levels in gestational diabetes mellitus. J Perinat Med. 2013;41:375-80.

48 Wu L, Fang J, Chen L, Zhao Z, Luo Y, Lin C, Fan L. Low serum adropin is associated with coronary atherosclerosis in type 2 diabetic and non-diabetic patients. Clin Chem Lab Med. 2014;52:751-8.

49 Beigi A, Shirzad N, Nikpour F, Nasli Esfahani E, Emamgholipour S, Bandarian F. Association between serum adropin levels and gestational diabetes mellitus; a case-control study. Gynecol Endocrinol. 2015;31:939-41.

\section{Submit your next manuscript to BioMed Central and we will help you at every step:}

- We accept pre-submission inquiries

- Our selector tool helps you to find the most relevant journal

- We provide round the clock customer support

- Convenient online submission

- Thorough peer review

- Inclusion in PubMed and all major indexing services

- Maximum visibility for your research

Submit your manuscript at www.biomedcentral.com/submit

C) Biomed Central 